Journal of Molecular Structure, 24 (1975) 222-225

C) Elsevier Scientific Publishing Company, Amsterdam - Printed in The Netherlands

\title{
THE MICROWAVE SPECTRUM OF METHANE SULPHONYL CHLORIDE
}

B. P. VAN EIJCK, A. J. KORTHOF AND F. C. MIJLHOFF"

Laboratorium voor Kristalchemie der Rijksuniversiteit, Padualaan 8, Utrecht, The Netherlands.

(Received 25 February 1974)

ABSTRACT

The microwave spectrum of $\mathrm{SO}_{2} \mathrm{ClCH}_{3}$ was observed by means of Stark modulation and double resonance modulation. Rotational constants, centrifugal distortion parameters and nuclear quadrupole coupling constants are reported for the ${ }^{35} \mathrm{Cl}$ and ${ }^{37} \mathrm{Cl}$ species. An unambiguous choice between three possible electron diffraction structures could be achieved by the determination of the $\mathrm{O}-\mathrm{O}$ distance.

\section{INTRODUCTION}

The molecular structure of methane sulphonyl chloride $\left(\mathrm{SO}_{2} \mathrm{ClCH}_{3}\right)$ has been studied by electron diffraction by Hargittai and Hargittai [1]. These investigators found three molecular structures differing largely in the $\mathrm{O}-\mathrm{S}-\mathrm{O}$ angle and all equally acceptable as far as least squares fit criteria are concerned. One solution, however, was preferred on the grounds of vibiational amplitudes and trends in analogous compounds. As suggested by Dr. I. Hargittai, we checked the accuracy of the proposed structure by microwave spectroscopy.

MICROWAVE SPECTRA

The spectrum $(22-40 \mathrm{GHz})$ was recorded at room temperature with a conventional $10 \mathrm{kH} z$ Stark-modulated instrument at pressures of $0.005-0.02 \mathrm{~mm} \mathrm{Hg}$. The accuracy of frequency measurements was about $0.1 \mathrm{MHz}$, and better for strong lines with well resolved quadrupole components.

* Permanent address: Gorlaeus Laboratoria der Rijksuniversiteit, P.O. Box 75, Leiden, The Netherlands. 
The spectrum was dominated by many strong $b$-type $J_{K, J-K} \rightarrow J_{K+1, J-K-1}$ and $J_{K, J-K+1} \rightarrow J_{K+1, J-K}$ transitions. No $a$-type transitions could be observed, and only a few $b$-type $J \rightarrow J+1$ transitions, relatively weak and much affected by quadrupole splitting, occur in the spectrum. Hence for some time the complete determination of the rotational constants was prevented. This problem was solved with aid of double resonance, by applying an on-off modulated strong microwave field in a frequency region where the $2_{21}-3_{30}$ transition was expected, and observing the modulated absorption around the frequency where the $3_{30}-4_{41}$ transition was calculated to be for each pump frequency. In this way a double resonance was detected after scanning a $300 \mathrm{MHz}$ region in $10 \mathrm{MHz}$ steps with the pump klystron. After that, more R-type transition frequencies were measured with the double resonance method; most of these lines were almost invisible in the Stark spectrum because of the large number of much stronger Q-type transitions.

\section{RESULTS}

A few $J \rightarrow J+1$ lines could be resolved in four quadrupole components; the other lines were either unresolved or split in symmetric doublets. We studied 39

TABLE

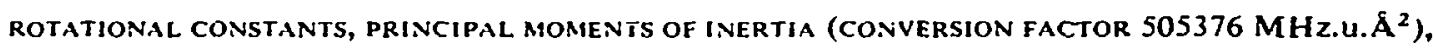
CENTRIFUGAL DISTORTION PARAMETERS AND NUCLEAR QUADRUPOLE COUPLING CONSTANTS FOR METHANE SULFONYL CHLORIDE

The number of transitions $N$ and the maximum value of $J$ are given for both $R$-type and Q-type transitions.

\begin{tabular}{|c|c|c|}
\hline & $\mathrm{SO}_{2}{ }^{35} \mathrm{ClCH}_{3}$ & $\mathrm{SO}_{2}{ }^{37} \mathrm{ClCH}_{3}$ \\
\hline$A(\mathrm{MHz})$ & $4636.421 \pm 0.005$ & $4636.319 \pm 0.008$ \\
\hline$B(\mathrm{MHz})$ & $2787.938 \div 0.005$ & $2714.847 \pm 0.006$ \\
\hline$C(\mathrm{MHz})$ & $2682.889 \div 0.005$ & $2615.081 \pm 0.011$ \\
\hline$I_{a}\left(\mathrm{U} . \mathrm{A}^{2}\right)$ & 109.0013 & 109.0037 \\
\hline$I_{b}\left(u \cdot \AA^{2}\right)$ & 181.2723 & 186.1527 \\
\hline$I_{c}\left(u \cdot \AA^{2}\right)$ & 188.3701 & 193.2544 \\
\hline$I_{a} \div I_{b}-I_{c}\left(\mathrm{u} . \AA^{2}\right)$ & 101.9035 & 101.9020 \\
\hline$\Delta,(H z)$ & $468 \pm 20$ & $457 \pm 30$ \\
\hline$\Delta_{J K}(\mathrm{~Hz})$ & $1894 \div 2$ & $1836 \pm 8$ \\
\hline$\Delta_{N}(H z)$ & $-1425 \pm 23$ & $-1426 \pm 50$ \\
\hline$\delta,(\mathrm{Hz})$ & $13.6 \pm 0.2$ & 13.6 (ass.) \\
\hline$\delta_{k}(\mathrm{~Hz})$ & $-1041 \pm 8$ & -1041 (ass.) \\
\hline$\varkappa_{a a}(\mathrm{MHz})$ & $-65.7 \pm 0.4$ & $-51.6 \div 0.5$ \\
\hline$\chi_{\Delta b}(\mathrm{MHz})$ & $29.9 \div 0.3$ & $23.8 \pm 0.4$ \\
\hline føce $(\mathrm{MHz})$ & $35.8 \pm 0.3$ & $27.8 \pm 0.4$ \\
\hline$\eta$ & $0.079 \pm 0.006$ & $0.068 \pm 0.008$ \\
\hline$N(\mathrm{R})$ & 8 & 7 \\
\hline$J_{\max }(R)$ & 13 & 13 \\
\hline$N(Q)$ & 47 & 18 \\
\hline$J_{\max }(Q)$ & 58 & 32 \\
\hline
\end{tabular}


transitions (91 quadrupole components) for $\mathrm{SO}_{2}{ }^{35} \mathrm{ClCH}_{3}$ and 21 transitions (54 quadrupole components) for $\mathrm{SO}_{2}{ }^{37} \mathrm{ClCH}_{3}$. By a least squares procedure the quadrupole coupling constants and the centre frequencies were then calculated, the respective r.m.s. deviations being 91 and $96 \mathrm{kHz}$. The transition frequencies thus obtained were combined with data for unresolved lines to obtain rotational constants and centrifugal distortion parameters [2]; in this calculation the r.m.s. deviation was $82 \mathrm{kHz}$ for both isotopic species. The measured frequencies are available on request; the molecular parameters are reported in Table 1.

The quadrupole coupling constants have, within experimental error, the correct ${ }^{35} \mathrm{Cl} /{ }^{37} \mathrm{Cl}$ ratio. Assuming that the principal quadrupole axis coincides with the $\mathrm{S}-\mathrm{Cl}$ bond, which makes an angle of only $4.6^{\circ}$ with the $a$-axis [1], we note a slight deviation from a cylindrically symmetric electron distribution about this axis. This is expressed by the parameters $\eta$ (Table 1 ), which agree well with the values $\eta=0.065 \pm 0.006$ for $\mathrm{SO}_{2}{ }^{35} \mathrm{ClF}$ and $\eta=0.066 \pm 0.012$ for $\mathrm{SO}_{2}{ }^{37} \mathrm{ClF}$ [3].

As in $\mathrm{SO}_{2} \mathrm{FCH}_{3}$ [4], no splittings due to internal rotation of the $\mathrm{CH}_{3}$ group were observed. Calculation shows that therefore $V_{3}$ must be at least $2 \mathrm{kcal} \mathrm{mol}^{-1}$.

\section{MOLECULAR STRUCTURE}

The $a$-substitution coordinate of the $\mathrm{Cl}$ atom is $1.5769 \pm 0.0001 \AA$; the $b$ coordinate is too small $(0.036 \AA)$ to be reliable. The $\mathrm{O}-\mathrm{O}$ distance can be found from the relation

$$
I_{a}+I_{b}-I_{c}=m_{\mathrm{O}} r_{\mathrm{OO}}^{2}+m_{\mathrm{H}} r_{\mathrm{HH}}^{2}=101.90 \mathrm{u} . \AA^{2}
$$

If we take $r_{\mathrm{HH}}=1.82 \AA$ [1] with the wide error limit of $0.05 \AA$, we find $r_{\mathrm{OO}}=2.483$ $\pm 0.003 \AA$. With the aid of the $S-O$ distance of $1.424 \pm 0.003 \AA$ [1] we calculate the $\mathrm{O}-\mathrm{S}-\mathrm{O}$ bond angle to be $121.3^{\circ} \pm 0.6^{\circ}$. Apart from improving the accuracy $\left(120.8^{\circ} \pm 2.4^{\circ}\right.$ in electron diffraction) this value confirms that M. and I. Hargittai have proposed the correct model out of the three possibilities. Also, the $\mathrm{Cl}$ position in the electron diffraction model $(a=1.583 \AA, b=0.047 \AA$ ) agrees fairly well with our result; the same applies to the inertial moments which differ less than $0.6 \%$ from the microwave data. No better agreement could be expected in view of the error limits of the electron diffraction parameters and, here less important, the neglect of vibrational corrections.

\section{ACKNOWLEDGEMENTS}

We wish to thank Dr. István Hargittai for his gift of the sample and for his interest in our work. We are also grateful to Dr. H. A. Dijkerman for his aid in the double resonance experiments and to Drs. G. de With who made the initial recordings of the spectrum. 


\section{REFERENCES}

I M. Hargittai and I. Hargittai, J. Chem. Phys., 59 (1973) 2513.

2 J. K. G. Watson, J. Chem. Phys., 46 (1967) 1935.

3 C. W. Holt and M. C. L. Gerry, Chem. Phys. Lett., 9 (1971) 621.

4 E. J. Jacob and D. R. Lide Jr., J. Chem. Phis's., 54 (1971) 4591. 